\title{
BER Analysis Of BPSK, QPSK, 16-QAM \& 64-QAM Based OFDM System Over Rayleigh Fading Channel
}

\author{
Pavan Kumar ${ }^{1}$, Amita Kumari ${ }^{2}$ \\ ${ }^{I}$ Electronics \& Communication Engineering Department MUIT, Lucknow India \\ ${ }^{2}$ Electronics \& Communication Engineering Department MUIT, Lucknow India
}

\begin{abstract}
Orthogonal frequency-division multiplexing (OFDM) is a method of encoding digital data on multiple carrier frequencies. The data are sent over parallel sub-channels with each sub-channel modulated by a modulation scheme such as BPSK, QPSK, 16 QAM etc. The advantage of OFDM is its ability to cope with severe channel conditions compared to a single carrier modulation scheme but still maintaining the data rates of a conventional scheme with the same bandwidth. Furthermore, channel equalization is simplified because OFDM may be viewed as using many slowly modulated narrow band signals rather than one rapidly modulated wide band signal. Also, the low symbol rate naturally makes the use of guard interval between symbols reducing ISI. Orthogonal Frequency Division Multiplexing has become one of the mainstream physical layer techniques used in modern communication systems. In this paper the OFDM system for a number of subcarriers is simulated and sent over Rayleigh fading channel. The performance of OFDM along with various modulation schemes in the Rayleigh Fading Channel environment is observed and presented in this paper.
\end{abstract}

Keywords: OFDM, Modulation, BPSK, QPSK,QAM, Bit Error Rate(BER), Rayleigh Fading Channel

\section{Introduction}

Multi band OFDM Ultra Wide Band transmits data simultaneously over multiple carriers spaced apart at precise frequencies on more than one band. OFDM signal needs precisely overlapping but non-interfering carriers, and achieving this precision requires the use of a real-time Fourier transform, which became feasible with improvements in Very Large-Scale Integration (VLSI). Basically, multi band OFDM system provides timedomain diversity by time-domain symbol spreading technique and frequency-domain diversity by transmitting OFDM symbols in different sub-bands. Fast Fourier Transform algorithms provide nearly 100 percent efficiency in capturing energy in a multi-path environment, while only slightly increasing transmitter complexity. Beneficial attributes of multi band OFDM include high spectral flexibility and resiliency to RF interference and multi-path effects. Although a wide band of frequencies could be used from a theoretical viewpoint, certain practical considerations limit the frequencies that are normally used for multi band OFDM ultra wide band communication. Limiting the upper bound simplifies the design of the radio and analogue front end circuitry as well as reducing interference with other services.An OFDM system offers inherent robustness to multi-path dispersion with a low-complexity receiver. Adding a Cyclic Prefix (CP) forces the linear convolution with the channel impulse response to resemble a circular convolution. A circular convolution in the time domain is equivalent to a multiplication operation in the frequency domain. An important stage in the implementation of OFDM is the modulation of the baseband signal along with the various subcarriers. Baseband signal cannot be transmitted without modulation. Information of baseband signal is transmitted in the way that parameter of high frequency carrier wave, such as amplitude or phase, is modulated by baseband signal, hence conveys the information that can be restored to original signal at the receiver. Selection of proper modulation scheme is essential to communication system design. In this research paper, we present the analysis of various modulation schemes in respect to their performance in terms of BER. For this research work, we have taken BPSK, QPSK, 16 QAM, 64 QAM as the modualtion schemes and have compared the performance in terms of BER and power spectral density, in a Rayleigh Fading Channel. The following sections present the Literature Review, communication models used and the result and analysis.

\section{Literature Review}

In [1] Mohammed S. Akhoirshida et.al have analyzed the performance of OFDM- BPSK, system by using forward error correcting codes(convolutional, reed Solomon as well as concatenated coding) schemes that are used to encode the data stream in wireless communications AWGN channel has been reported here. The authors have presented OFDM for wireless communications .They address basic OFDM and related modulations, as well as techniques to improve the performance of OFDM for wireless communications (OFDM). The various simulations have been done to find out the best BER performance of each of the Convolutional and Reed-Solomon codes and used these best outcomes to model the RS-CC concatenated codes. 
In [2], Jun Lu, has considered a space-time coded (STC) orthogonal frequency-division multiplexing (OFDM) system with multiple transmitter and receiver antennas over correlated frequency and time-selective fading channels. It is shown that the product of the time-selectivity order and the frequency-selectivity order is a key parameter to characterize the outage capacity of the correlated fading channel. They have observed that STCs with large effective lengths and ideal built-in interleavers are more effective in exploiting the natural diversity in multiple antenna correlated fading channels.

In [3], Y.J Ryu et.al have proposed A timing offset estimator robust to a Rayleigh fading channel and a fast Fourier transform (FFT) window selection algorithm that can remove inter symbol interference (ISI). The use of amplitude information in the proposed algorithm means that the influence of distorted pilots due to fading becomes insignificant when estimating a timing offset. The proposed FFT window selection algorithm based on the cyclic extension property of the OFDM structure can remove the ISI problems caused by multipath fading channels, thereby significantly improving the receiver performance without any additional computational requirements. The paper uses the DVB-T standard to evaluate the algorithms.

In [4-7] and [10], Nagakami m-fading channel and the various methods to improve BER performance in it, have been presented. Error rates of orthogonal frequency-division multiplexing (OFDM) signals in multipath slow fading Nagakami-m fading channels are considered. The exact probability density function of a sum of Nagakami-m random phase vectors is used to derive a closed-form expression for the error rates of OFDM signals. In [6], M.K.Mishra et. al, have proposed that average BER expressed in terms of the higher transcendental function such as the confluent hyper geometric functions. The numerical results show that depending on the number of channel taps, the BER performance may degrade with increasing values of Nakagami-m fading parameters.

Zhangyong Ma, has proposed a novel scheme for joint carrier frequency offset and channel estimation in a Rayleigh flat-fading channel in [8]. A simple time-domain weighted algorithm based channel estimation with pilot-data multiplexed scheme is investigated. The simulation results show that the proposed methods achieve more accurate estimation of the CFO and the time-variant channel parameter than the conventional schemes, and the system performance is improved dramatically.

SONG Lijun et. al. have studied the performance of frequency domain differential demodulation(FDDD). The bit error rate (BER) performance of for orthogonal frequency division multiplexing system has been evaluated by computer simulation and compared with the time domain differential demodulation(TDDD). The results indicate that the performance of FDDD is better than that of TDDD, and the lower band of BER in the former is lower than that of the latter[9].

\section{Modulation Schemes}

Modulation is the process of varying some characteristic of a periodic wave with an external high frequency signal. It is utilized to send an information bearing signal over long distances. These high frequency carrier signals can be transmitted over the transmitted lines or air and are capable of traveling long distances. The carrier signal can be an electrical current, a radio or microwave frequency or light. The process of extracting the information at the receiving end is called demodulation. The characteristics (amplitude, frequency, or phase) of the carrier signal are varied in accordance with the information bearing signal. This information bearing signal is also known as the modulating signal. This modulating signal is a slowly varying signal as opposed to the rapidly varying carrier frequency.

\section{M-PSK}

The idea of Phase-shift keying (PSK) modulation scheme is that information of baseband signal is conveyed by changing of carrier wave's phase. Family of coherent M-PSK includes BPSK, QPSK, 8PSK and 16PSK, where BPSK, 8PSK and 16PSK are in discuss in this section.BPSK is a binary digital modulation scheme, which is also the simplest form of M-PSK. Binary data (" 0 " and " 1 ") are represented by two carrier waves with phases of 0 and 1 respectively, which has the following form:

$S_{0}(t)=A \cos 2 \pi f_{c} t, \quad 0 \leq t \leq T_{b}$

where $\mathrm{A}$ is a constant amplitude, $\mathrm{fc}$ is carrier frequency and $\mathrm{Tb}$ is the bit duration.Suppose bit energy is denoted as Eb, expressed as:

$$
\begin{aligned}
& S_{0}(t)=\sqrt{\frac{2 E_{b}}{T_{b}}} \cos 2 \pi f_{c} t, \quad 0 \leq t \leq T_{b} \\
& S_{1}(t)=-\sqrt{\frac{2 E_{b}}{T_{b}}} \cos 2 \pi f_{c} t, \quad 0 \leq t \leq T_{b}
\end{aligned}
$$




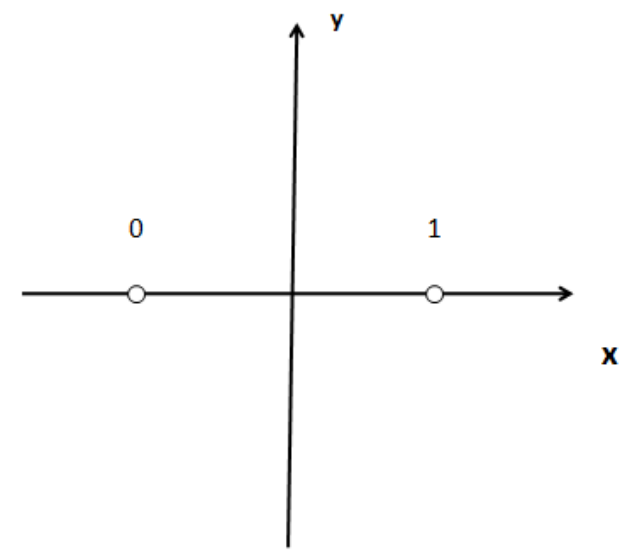

Figure 1: Constellation diagram of BPSK

\section{M-QAM}

Quadrature amplitude modulation (QAM) is the most commonly used type of modulation technique in OFDM [7]. As a multi-level modulation scheme, QAM modulation scheme acquires higher data rate, that is, higher bandwidth efficiency, by sacrificing power utilization. It implies that higher SNR is required for QAM if we intend to maintain low bit error rate.QAM can be regarded as the combination of two modulations on in-phase (real) and quadrature (imaginary) branches since the carrier wave experiences amplitude as well as phase modulation [10].

The constellation of 16QAM is shown in figure 1:

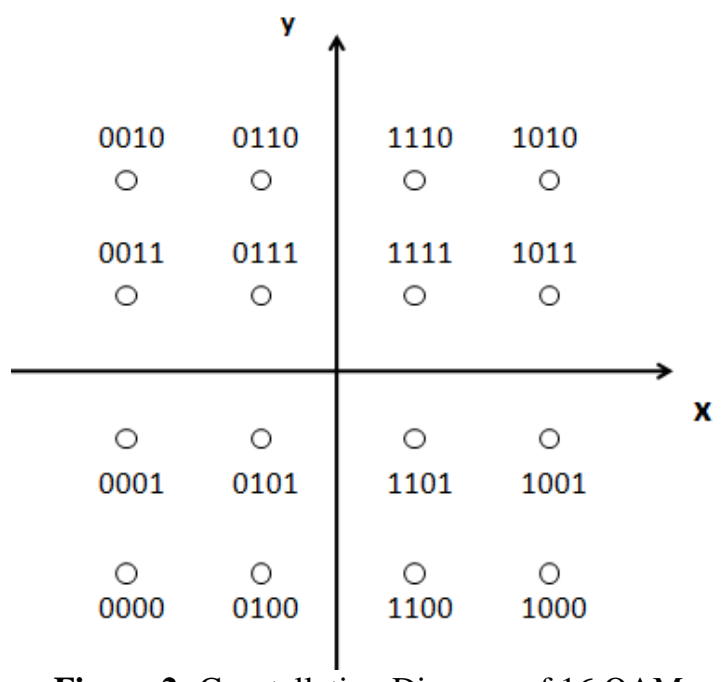

Figure 2: Constellation Diagram of 16 QAM

In QAM modulation scheme, the in-phase and quadrature components are independent. Herein, the probability of correct detection ia can be written as:

$$
P_{c}=\left(1-P_{e}^{\prime}\right)
$$

where $\mathrm{P}_{\mathrm{e}}$ ' is the probability of symbol error for either in-phase component or quadrature component. Thus, the probability of symbol error for $16 \mathrm{QAM}$ is given by:

$$
P_{e}=1-P_{c}
$$

The probability of error is given as:

$$
P_{e}=1-\left[1-\frac{2(\sqrt{M}-1)}{\sqrt{M}} Q\left(\sqrt{\frac{3 E_{a v}}{2(M-1) N_{0}}}\right)\right]^{2}, \quad M=16
$$

There are four bits per symbol for 16 QAM modulation scheme. The bit error probability for 16 QAM is denoted as: 


$$
P_{b}=\frac{3}{4} Q\left(\sqrt{\frac{4 E_{b}}{5 N_{0}}}\right)-\frac{9}{16} Q^{2}\left(\sqrt{\frac{2 E_{b}}{7 N_{0}}}\right)
$$

System modulated by $M$-ary QAM scheme acquires higher data transmission rate by suffering BER degradation.

\section{Channel modeling:}

Analysis and modeling of radio propagation is a most important issue in wireless communication. Only by properly measuring the characteristic of fading channel, communication system can be correctly developed. The various kinds of channel model are discussed below:

\section{Small Scale Fading}

There are generally two types of fading in wireless communication: large scale and small scale fading. Large scale fading, which is the major concern in microwave communications, is mainly caused by long propagation distance and large obstacles like mountains and buildings, where signal power attenuates with the increase of distance. Other situations, such as the change of climate, also cause large scale fading. The affect of large scale fading is not taken into consideration when the area of communication is relatively small.

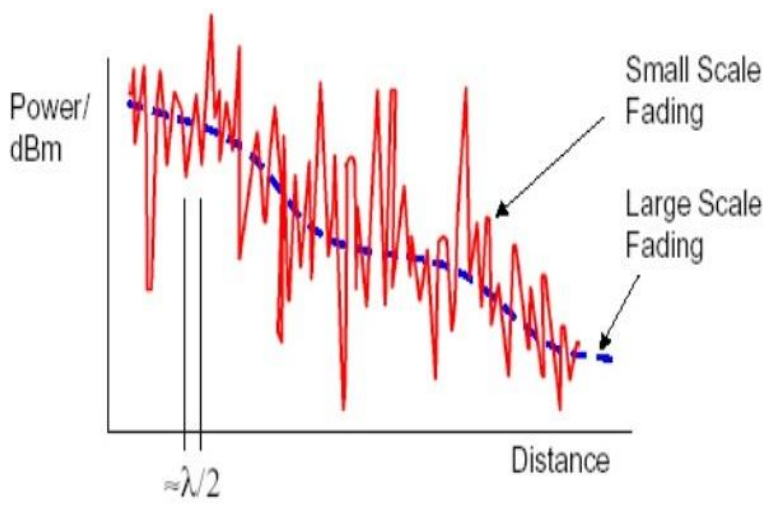

Figure 3: Small Scale vs Large Scale Fading

\section{Typical Small Scale Fading Models}

In this section, two typical small-scale fading channel models, Rayleigh and Rician, are presented and investigated. Modeling of radio propagation is essential to wireless communication systems since it enables one to adopt appropriate means to reduce signal attenuation and distortion. Rician and Rayleigh models are most commonly used to describe wireless propagation fading channel, especially mobile communication channel.

Rayleigh fading environment is characterized by many multipath components, each with relatively similar signal magnitude, and uniformly distributed phase, which means there is no line of sight (LOS) path between transmitter and receiver. Therefore, Rayleigh fading is often considered as a worst-case scenario for mobile communications within urban environments.

For Rayleigh fading channel, there exist a large number of multipath components, each with random amplitude and phase. According to central limit theorem, the real and imaginary components of the complex envelope comply with Gaussian distribution. Suppose $\mathrm{x}$ and y denoted real and imaginary components respectively. The possibility density function (PDF) of the two is respectively expressed as:

$$
\begin{gathered}
p_{x}(x)=\frac{1}{\sigma \sqrt{2 \pi}} e^{\frac{-x^{2}}{2 \sigma^{2}}} \\
p_{y}(y)=\frac{1}{\sigma \sqrt{2 \pi}} e^{\frac{-y^{2}}{2 \sigma^{2}}}
\end{gathered}
$$

where $\sigma$ represents the standard deviation of the envelope amplitude (also known as the rms value of the envelope). Since both random variables are independent and identically distributed, the joint distribution can be written as:

$$
p_{x y}(x, y)=p_{x}(x) \cdot p_{y}(y)=\frac{1}{2 \pi \sigma^{2}} e^{\frac{-\left(x^{2}+y^{2}\right)}{2 \sigma^{2}}}
$$


Therefore Probabilty Density Function:

$$
f_{R}(r)=\frac{r}{\sigma^{2}} \exp \left(\frac{-r^{2}}{2 \sigma^{2}}\right)
$$

\section{Rician Fading Model}

Rician fading model is another most commonly employed model, which is adopted when there is a dominant LOS path and a number of weak multipath components in propagation environment. In mobile communication, Rician fading model can be used when mobile station is moving across the open ground, such as suburb and rural area, where LOS signal can be received.

The fading amplitude $r$ th at the ith time instant can be represented as:

$$
r_{i}=\sqrt{\left(x_{i}+V\right)^{2}+y_{i}^{2}}
$$

Where $\mathrm{K}$ is the amplitude of the specular component, $\mathrm{x}^{\mathrm{a}}$ and $\mathrm{y}^{\mathrm{a}}$ are samples of zero-mean stationary Guassian random processes each with variance $\sigma 02$. The ratio of specular to diffuse energy is known as Rician K-factor, which is given by:

$$
K=V^{2} / 2 \sigma_{0}^{2}
$$

$\mathrm{K}$-factor reflects extent of LOS signal in Rician fading. If $\mathrm{K}=\infty$, the LOS signal is so strong that diffuse waves can be regarded as white Gaussian noise. The fading behavior will follow Gaussian distribution. On the other hand, there is no LOS path in propagation channel, and the fading behavior will show the characteristic of Rayleigh fading. The Rician PDF is shown as below:

$$
f_{\text {rice }}(r)=\frac{r}{\sigma_{0}^{2}} \exp \left[-\frac{r^{2}+V^{2}}{2 \sigma^{2}}\right] I_{0}\left[\frac{r V}{\sigma_{0}^{2}}\right]
$$

Where $\mathrm{I} 0$ is 0th order modified Bessel function of the first kind.

\section{System Model}

The system architecture which has been used for this research work is discussed in this chapter. The below figure shows the block diagram of the model used for simulation in this research work.

\section{Input:}

We used a random bit generator to generate a random bit stream. The output is a 1 dimensional array of bits. We then perform a serial-to-parallel conversion sending the bits on parallel streams each representing a subcarrier.

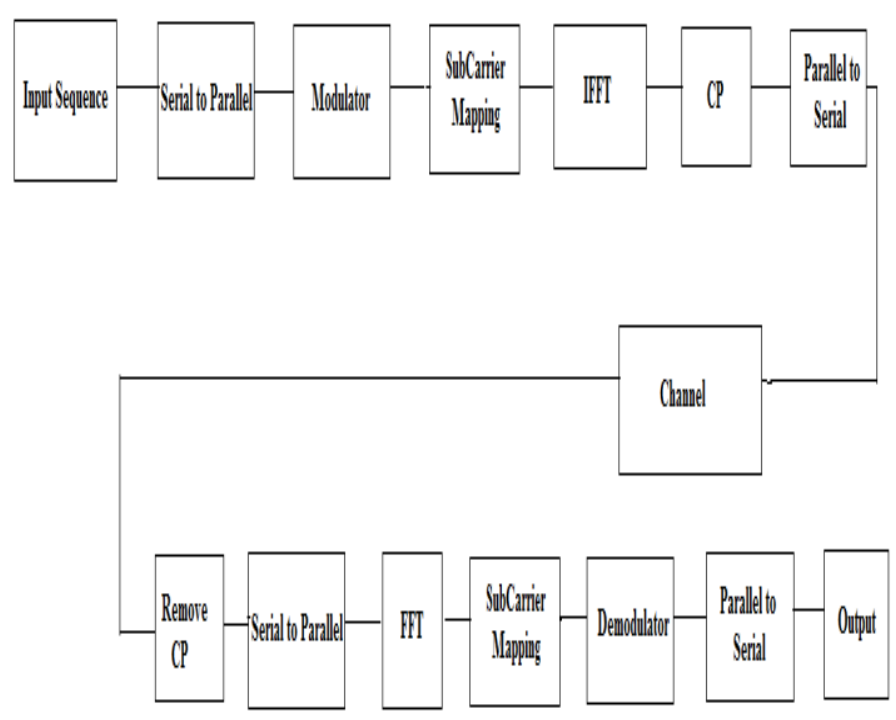

Figure 4: OFDM Architecture 


\section{Series to Parallel:}

The series to parallel conversion is done to allot the bitstream generated in previous step to the various subcarriers. The number of subcarriers is taken to be 512. Thus the input bitstream is divided into 512 parallel bitstreams.

\section{Modulator:}

Variousmodulation schemes have been used on all the subcarriers. The modulation schemes have been used and the results have been simulated. BPSK, QPSK, 16QAM, 64 QAM are the various modulation schemes which have been used.

\section{Sub Carrier Mapping:}

The mapping of sub carriers is the next step to align the bit stream over the subcarriers.

\section{IFFT:}

We then perform the IFFT of all the parallel data streams together ensuring orthogonally between the subcarriers and the conversion of symbols to time domain. By orthogonality, it is meant that all the subcarriers on which the data has been sent overlap each other in such a way that they don't interfere with one another and ensure minimum bandwidth usage. IFFT for a set of $\mathrm{N}$ complex data points from $\mathrm{N}$ orthogonal parallel streams is given by the formula.

$$
\mathrm{x}(\mathrm{n})=\frac{1}{\mathrm{~N}} \sum_{\mathrm{k}=0}^{\mathrm{N}-1} \mathrm{X}(\mathrm{k}) \cdot \mathrm{e}^{\mathrm{j}\left(\frac{2 \pi}{\mathrm{N}}\right) \mathrm{nk}} ;(\mathrm{n}=0,1, \ldots \mathrm{N}-1)
$$

Where, $\mathrm{X}(\mathrm{k})$ is a complex frequency domain data sent on subcarriers of frequency $\mathrm{k} / \mathrm{N}, \mathrm{k}=0,1, \ldots, \mathrm{N}$ $\mathrm{k} / \mathrm{N}$ term is orthogonal to every other value of $\mathrm{k} / \mathrm{N}$

\section{Cyclic prefix:}

Cyclic prefix addition is the next step of the procedure. The term cyclic prefix refers to the prefixing of a symbol with a repetition of the end. Although the receiver is typically configured to discard the cyclic prefix samples, the cyclic prefix serves two purposes:

- As a guard interval, it eliminates the inter symbol interference from the previous symbol.

- As a repetition of the end of the symbol, it allows the linear convolution of a frequency-selective multipath channel to be modeled as circular convolution, which in turn may be transformed to the frequency domain using a discrete Fourier transform. This approach allows for simple frequency-domain processing, such as channel estimation and equalization.

\section{Parallel to Serial:}

The cyclic prefixed bit stream is now converted back to serial bit stream to be transmitted over the channel.

\section{Channel:}

Rayleigh channel model has been used as the fading channel for this research work. The Rayleigh channel is implemented using the built-in function.

\section{Receiver Side}

\section{Remove CP:}

The cyclic prefix added at the time of transmission is removed in this step. This is the first step at the receiving end.

\section{Serial to parallel:}

The serial bit stream is converted to parallel data and is mapped onto the respective subcarriers.

FFT:

Fast Fourier Transform is used to convert the signal to Fourier domain to make the analysis easier. Built in functions is used to convert the data stream into Fourier domain.

\section{Sub Carrier Mapping:}

The subcarrier mapping is used to map the subcarriers with the respective bit stream pattern on them.

\section{Demodulator:}

The demodulator demodulates the bit stream, so as to retrieve the original or baseband signal. According to the type of modulation, used the corresponding demodulation method is applied.

Parallel to Serial:

The parallel data is then converted back to serial bit pattern to retrieve the message which is the final output 
V. Simulation Results

\begin{tabular}{|l|c|}
\hline Parameter & Value \\
\hline Number of Subcarriers & 512 \\
\hline FFT Length & 512 \\
\hline Bandwidth & $5 \times 10^{6}$ \\
\hline Sampling Frequency & $2 \times B W$ \\
\hline Cyclic Pad Length & 64 bits \\
\hline Modulation Technique & BPSK,QPSK,16QAM,64QAM \\
\hline
\end{tabular}

Table 1: Simulation Parameters

Simulation Results and Graphs:

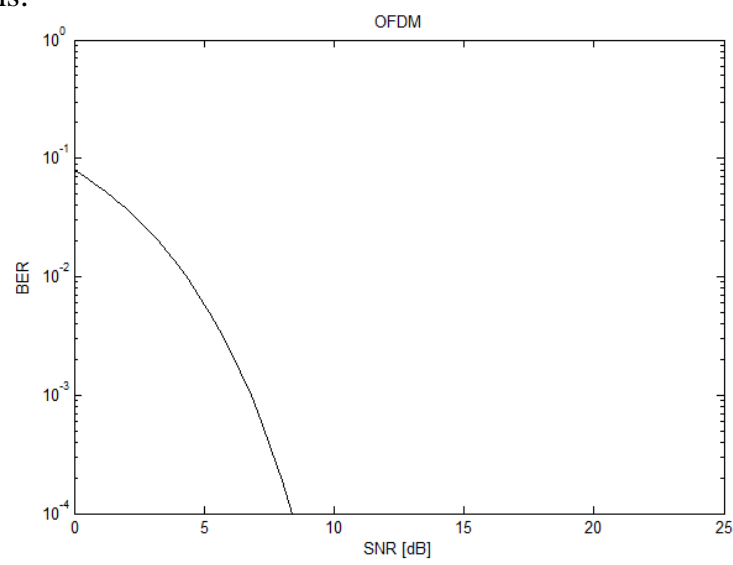

Figure 5: BPSK BER vs SNR curve

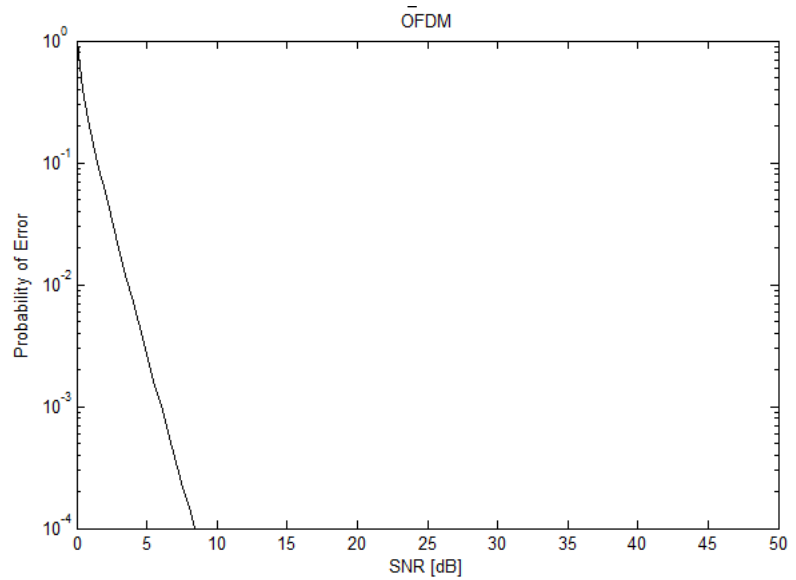

Figure 6: Probability of Error

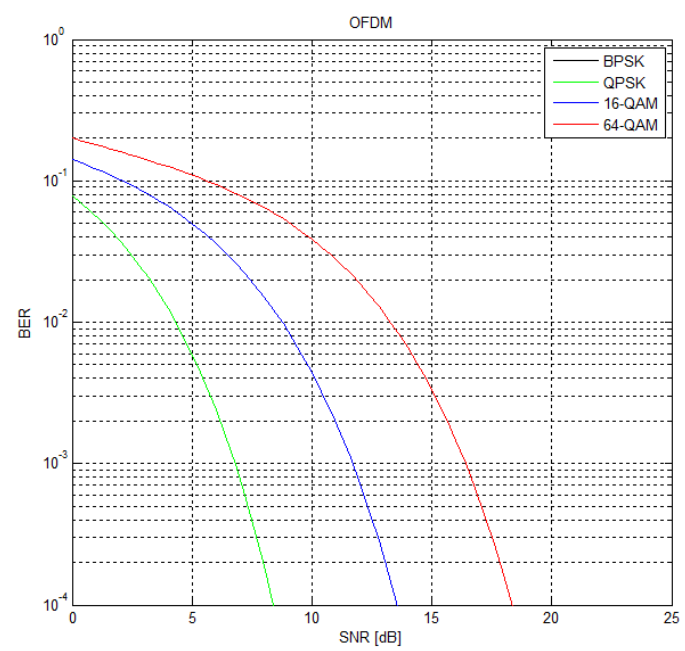


Figure 7: BER vs SNR curves

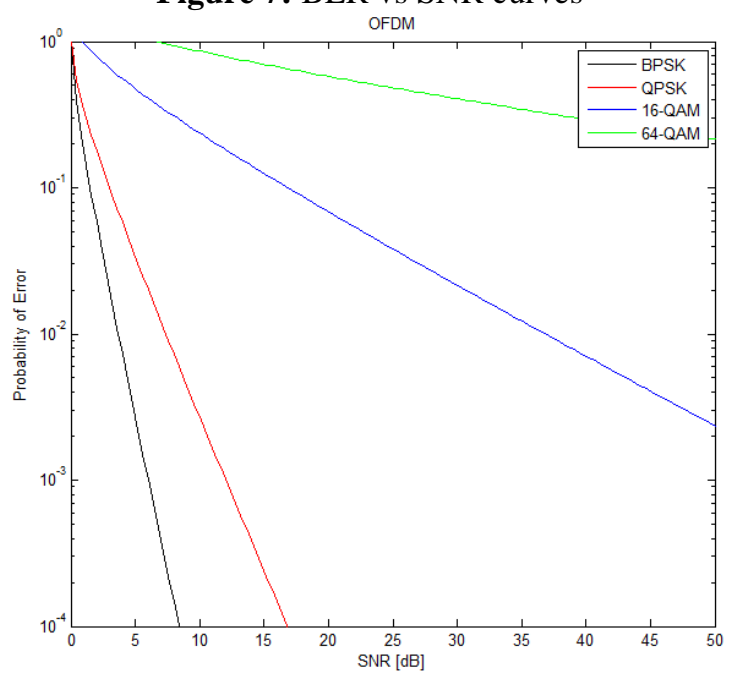

Figure 8: Probability of Error

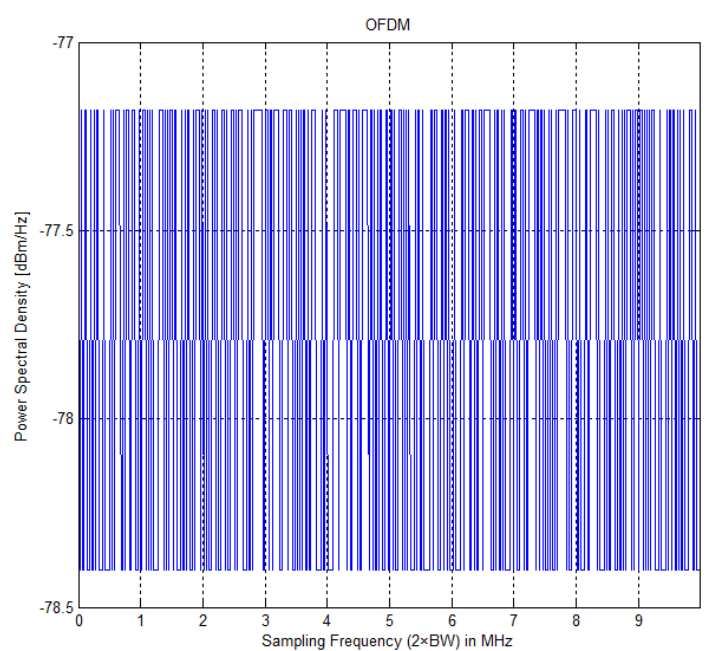

Figure 9: PSD Vs sampling Frequency

Figure 7 shows the BER vs SNR plot for various modulation techniques which have been used in this research work. The 64 QAM modulation techniques show better performance over Rayleigh Channel.

Figure 8 shows the Probability of error graph against SNR. This graph shows the bandwidth performance of OFDM under Rayleigh channel for various modulation techniques. There always exists a tradeoff between the bandwidth efficiency and BER vs SNR performance.

Figure 9 shows the Power Spectral density is plotted against sampling frequency. The power Spectral density graph shows the various subcarriers.

\section{Conclusion}

In this research work, the OFDM model of Wireless Communication is implemented and a number of modulation schemes are used viz. BPSK, QAM, QPSK etc. The various performance parameters like BER, SNR etc are to be evaluated. The channel used is Rayleigh fading Channel, for this research. The research work is intended to study and analyze the performance of OFDM technique under various modulation schemes. The MATLAB software which will be used for the implementation of this research work contains a wide range of functions to study, analyze and simulate the various kinds of communications system. This research work studies the vagaries of a Rayleigh fading channel, which is the most widely researched models of fading channels.

The OFDM system was simulated with an attempt to single out the performance of various modulation techniques under Rayleigh Channel environment. The key inferences from this research work are that BER is the key parameter for indicating the system performance of any data link. The BER vs SNR plot for various modulation techniques shows that for higher order modulation schemes the BER increases with increasing SNR. On the other hand, the lower order modulation schemes (BPSK and QPSK) experience less BER at receiver thus lower order modulations improve the system performance in terms of BER and SNR. 


\section{References}

[1]. Mohammed S. Akhoirshida and Mustafa M. Matalgah--BER Performance Analysis of Interference-Limited BPSK Cooperative Communication Systems with Cochannel Interference in Nakagami-m Fading Channels, PAWR 2013,IEEE.

[2]. Jun Lu, ThiangTjhung,Fumiyuki Adachi and Cheng Li Huang, -BER performance of OFDM-MDPSK system in Frequency Selective RicianFading with Diversity Reception, IEEE Trans. On Vehicular Technology, vol. 49, no. 4, pp. 1216-1225, July 2000.

[3]. Young Jae Ryu and Dong Seog Han, - Timing phase estimator overcoming Rayleigh Fading For OFDM systems, IEEE Proc., pp. 66- 67.

[4]. M. Nakagami, - The m-distribution-A general formula of intensity distribution of rapid fading,\| in Statistical Methods in Radio Wave Propagation, W. C. Hoffman, Ed. Elmsford, NY: Pergamon, 1960.

[5]. Zheingjiu Kang, Kung Yao, FlavioLorenzelli, - Nakagami-m Fading Modeling in the Frequency Domain for OFDM system analysis,\| IEEE Communication letters, vol. 7, no.10, pp. 484-486, Oct.2003.

[6]. Zhengdu,Julian Cheng and Norman c. Beaulieu, - Asymptotic BER performance of OFDM in Frequency Selective Nakagami-m Channels,ll IEEE Conference on Vehicular Technology, vol. 1, pp. 612-615,Sept. 2004.

[7]. Zhengdu,Julian Cheng and Norman c. Beaulieu, -Accurate Error Rate Performance Analysis of OFDM on Frequency SeelectiveNakagami-m Fadiing Channels,\|l IEEE Trans.oncommunications.vol. 54, no. 2, pp. 319-328, Feb. 2006.

[8]. Zhangyong Ma and Young-il- Kim, - A Novel OFDM receiver in Flat Fading Channel,\| IEEE Conference on advanced communication technology, ICACT, Vol.. 2, pp. 1052-54, 2005.

[9]. SONG Lijun, TANG Youxi, LI Shaoqian and HUANG Shunji, -BER Performance of Frequency Domain Differential Demodulation OFDM in Flat Fading Channel, IJ Journal of Electronic Science and Technology of China, Vol. 1, no. 1, Dec. 2003.

[10]. JyoteeshMalhotra, Ajay K. Sharma, R.S Kaler, - Investigation on First Order Performance Metrics in the Nakagami-m Fading Channel,\|In the proceeding of conference of Design Technique for ModerenElectonic Devices, VLSI and Communication Systems, 14th-15th May,2007.

[11]. E. Biglicri, J. Proakis and S. Shamai, "Fading channels: information-theoretic and communications aspects," IEEE Trans. Inform. Theory, vol. 44, no. 6, pp.2619-2692, Oct. 1998.

[12]. M. Patzold, U. Killat, F. Laue and Y. Li, "On the statistical properties of deterministic simulation models for mobile fading channels," IEEE Trans. Veh.Technol.,vol. 47, no. 1, pp. 254-269, Feb. 1998

[13]. J. Frolik, T. Weller, S. DiStasi and J. Cooper, "A compact reverberation chamber for hyper-Rayleigh channel emulation," IEEE Trans. Antennas propag., vol. 57, no. 12, pp. 3962-3968, Dec. 2009.

[14]. A. Henderson, G. Durgin and C. Durkin, "Measurement of small-scale fading distributions in a realistic $2.4 \mathrm{GHz}$ channel."

[15]. B. Ai, J. Ge, Y. Wang, S. Yang and P. Liu, "Decimal frequency offset estimation in COFDM wireless communications," IEEE Trans. Broadcasting, vol. 50, no. 2, pp. 154-158, Jun. 2004

[16]. S. Coleri, M. Ergen, A. Puri and A. Bahai, "Channel estimation techniques based on pilot arrangement in OFDM systems", IEEE Trans. Broadcasting, vol. 48, no.3, pp. 223-229, Sept. 2002.

[17]. W. C. Jakes, Ed., Microwave Mobile Communications, New York: IRRR Press, 1974

[18]. C. Xiao, Y. Zheng, N. Beaulieu, "Novel sum-of-sinusoids simulation models for Rayleigh and Rician fading channels", IEEE Trans. Wireless Commun., vol. 5, no. 12, pp. 3667-3678, Dec. 2006

[19]. E. Tufvesson, T. Maseng, "Pilot assisted channel estimation for OFDM in mobile cellular systems," in Proc. IEEE 47th Vehicular Technology Conference, Phoenix, USA, May 1997, pp. 1639-1643

[20]. J. Choi, Y. Lee, "Optimum pilot pattern for channel estimation in OFDM systems", IEEE Trans. Wireless Commun., vol. 4, no. 5, pp. 2083-2088, Sep. 2005

[21]. M. J. Fern'andez-GetinoGarc'1a, S. Zazo and J. M. P'aez-Borrallo, "Pilot patterns for channel estimation in OFDM," Electron.Lett., vol. 36, no. 12, pp. 1049-1050, June 2000.

[22]. M. Hsieh, C. Wei, "Channel estimation for OFDM systems based on comb-type pilot arrangement in frequency selective fading channels,” IEEE Trans. Consumer Electron., vol. 44, no. 1, pp. 217-225, Feb. 1998.

[23]. Y. Li, "Pilot-symbol-aided channel estimation for OFDM in wireless systems", IEEE Trans. Veh. Technol.,vol. 48, no. 4, pp. 12071215, Jul. 2000

[24]. J. Moon, S. Choi, "Performance of channel estimation methods for OFDM systems in a multipath fading channels," IEEE Trans. Consum.Electron., vol. 46, no. 1, pp. 161-170, Feb. 2000.

[25]. X. Dong, W. Lu and A. Soong, "Linear interpolation in pilot symbol assisted channel estimation for OFDM," IEEE Trans. Wireless Commun., vol. 6, no. 5, pp.1910-1920, May. 2007

Mrs. Amita is currently Assistant professor at Maharishi Institute of Information lucknow, India. She has

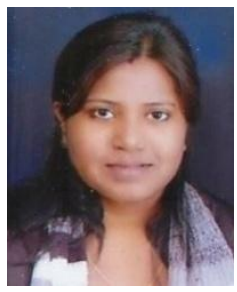
completed M.E from National Institute of Technical Teachers Training \& Research Chandigarh, India. She has completed B.Tech from Dr B S.B.R.A College of Agricultural Engineering and Technology, Etawah (U.P). She is having more than 6 years of teaching experience and published 3 international journals. Mrs Amita's interest areas are Image processing, VLSI, wireless and mobile communication and digital electronics.

Mr. Pavan kumar is currently pursuing his M,Tech from MUIT, Lucknow in microwave engineering . He has

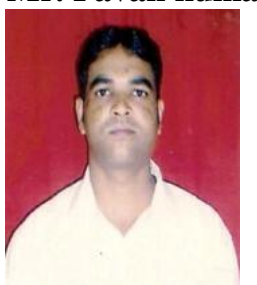
completed his B.Tech from SSITM Aligarh affiliated to UPTU. His interest areas are microwave engineering, digital electronics, analog and digital communication. 